\title{
Prevalence of Listeria monocytogenes and Escherichia coli O157:H7 in strawberries in Greece and performance evaluation of the culture media
}

\author{
Agni Hadjilouka, Valia Koubou, Spiros Paramithiotis*, Marios Mataragas, Eleftherios H. Drosinos \\ Laboratory of Food Quality Control and Hygiene, Department of Food Science and Human Nutrition, Agricultural University of Athens, Greece
}

\begin{abstract}
The aim of the present study was to determine the prevalence of $L$. monocytogenes and $E$. coli $\mathrm{O} 157: \mathrm{H} 7$ in strawberry samples by using established ISO protocols. Moreover, the performance of the substrates used for that purpose, i.e. ALOA and RAPID'L.mono regarding L. monocytogenes and Fluorocult E. coli O157:H7 and CT-SMAC regarding E. coli O157:H7 was evaluated and compared. In addition, direct detection by specific PCR after incubation of the enrichment broths was performed. Prevalence of both pathogens was estimated at 3.8\%. ALOA and RAPID'L.mono provided with the same results and thus their performance indices were identical. This was not the case regarding Fluorocult E. coli O157:H7 and CT-SMAC, in which, sensitivity and negative prediction value were optimal for both substrates but Fluorocult E. coli O157:H7 performed better in terms of specificity, positive prediction value and positive likelihood ratio. Finally, specific PCR performed after incubation of the enrichment broths is not recommended as an indicator for the presence of these pathogens.
\end{abstract}

\section{Introduction}

Listeria monocytogenes and Escherichia coli O157:H7 are considered as major foodborne pathogens since they have been implicated in a high number of outbreaks linked with food of both animal and plant origin [1-7]. In raw fruits and vegetables, prevalence of L. monocytogenes and E. coli $\mathrm{O} 157: \mathrm{H} 7$ has been reported to range from 0.7 to $36.8 \%$ [8-10] and from 3 to $18 \%[7,11]$, respectively.

In Greece, 84 confirmed cases of human listeriosis were reported during the decade 2004-2014 [12-15]. In addition, from 2004 to 2013, the mean annual number of cases was 7.30 and the mean annual notification rate was 0.65 cases per $1,000,000$ population [16]. Contrary to L. monocytogenes cases, the mean annual notification rate of EHEC infection for the period 2004-2015 was significantly lower, with 0.08 cases per 1,000,000 population. In total, 10 cases were reported for this period $[15,17,18]$. Regarding their prevalence in fruits and vegetables in Greece, it has been estimated at $6 \%$ and 3\% in cucumber for $L$. monocytogenes and E. coli $\mathrm{O} 157: \mathrm{H} 7$, respectively and at $7 \%$ in rocket for both pathogens [19].

Culture-dependent detection of foodborne pathogens, despite time-consuming is still the reference approach. In the case of $L$. monocytogenes and E. coli O157:H7 the chromogenic media most commonly used are Agar Listeria according to Ottaviani and Agosti (ALOA) and sorbitol MacConkey medium with cefixime and tellurite (CT-SMAC) [20,21]. However, their performance during routine microbiological analysis is not always perfect leading to over- or underestimation of prevalence. Indeed, differences in the performance of media used for the detection of L. monocytogenes and E. coli O157:H7 are very often reported $[19,22,23]$. It seems thus important to combine chromogenic media, in order to improve the accuracy of the analysis. The time-consuming nature of the analysis is very often managed by detection of the pathogens via specific PCR after incubation of the enrichment broths [24] and using the result of this analysis as an indication, especially in the case of products with short shelf-life.
The aim of this study was to determine the prevalence of $L$. monocytogenes and E. coli $\mathrm{O} 157: \mathrm{H} 7$ in strawberry samples by using ISO protocols, to evaluate and compare the chromogenic media ALOA and RAPID'L.mono used for L. monocytogenes and Fluorocult E. coli O157:H7 and CT-SMAC used for E. coli O157:H7 recovery and to compare these results with specific PCR performed after incubation of the enrichment broths.

\section{Materials and methods}

\section{Sample collection}

Samples of strawberries (Fragaria ananassa, $\mathrm{n}=26$ ), originating from different geographical districts of Greece, were purchased from supermarkets in the metropolitan area of Athens, Greece, during a survey in May 2012. Samples were transported to the laboratory at $4^{\circ} \mathrm{C}$ and analyzed the same day.

Detection, enumeration and confirmation of $L$. monocytogenes and E. coli O157:H7

Detection and enumeration of L. monocytogenes and E. coli O157:H7 was performed by classical microbiological techniques. Detection was also performed by specific PCR after incubation of the enrichment broths. In the first case detection and enumeration

Correspondence to: Spiros Paramithiotis. Laboratory of Food Quality Control and Hygiene, Department of Food Science and Human Nutrition, Agricultural University of Athens, Iera Odos 75 GR11855, Athens, Greece, Tel: +302105294705; E-mail: sdp@aua.gr

Key words: Listeria monocytogenes, Escherichia coli O157:H7, prevalence, strawberry

Received: November 27, 2017; Accepted: January 22, 2018; Published: January 25,2018 
of L. monocytogenes were carried out according to ISO 11290-1:1996 and ISO 11290-2:1998, respectively, by spreading on the selective chromogenic media Agar Listeria according to Ottaviani and Agosti [21] (ALOA) (Biolife, Milan, Italy) and RAPID'L.mono (Bio-Rad, Paris, France). Similarly, detection and enumeration of E. coli O157:H7 was performed according to ISO 16654:2001 with Fluorocult E. coli O157:H7 (Merck, Darmstadt, Germany) and sorbitol MacConkey medium with cefixime and tellurite (CT-SMAC) (Lab M, Bury, UK).

Confirmation of L. monocytogenes and E. coli O157:H7 identity was performed by both biochemical and molecular tests. At least five typical presumptive L. monocytogenes and E. coli O157:H7 colonies were randomly selected and isolated from the plates of each substrate. The biochemical tests for L. monocytogenes (mobility, hemolysis, and rhamnose and xylose fermentation) were conducted according to ISO 11290-1:1996. In the case of E. coli O157:H7, the E. coli O157:H7 latex test (Remel, Lenexa, KS, USA) was used according to the instructions of the manufacturer. Molecular identification was performed by PCR according to D'Agostino, et al. [25] and Gordillo, et al. [26] for L. monocytogenes and E. coli $\mathrm{O} 157: \mathrm{H} 7$, respectively. A presumptive L. monocytogenes or E. coli O157:H7 colony was considered positive when both biochemical and molecular tests verified the identity.

Specific PCR was applied as follows: $10 \mathrm{~mL}$ of each enrichment broth (i.e. half Fraser and full Fraser for L. monocytogenes and mTSB for E. coli O157:H7) after incubation were centrifuged (12.000 x $g, 4$ ${ }^{\circ} \mathrm{C}, 10 \mathrm{~min}$ ), the pellet was washed twice with sterile saline and DNA was extracted according to Hadjilouka, et al. [27]. The PCR protocols developed by D'Agostino, et al. [25] and Gordillo, et al. [26] for L. monocytogenes and E. coli O157:H7, respectively, were used for pathogen detection.

\section{Calculation of performance indices}

A sample was considered positive when the identity of at least one presumptive colony of L. monocytogenes or E. coli O157:H7 recovered from any of the media was confirmed. The parallel use of two selective media resulted in a $2 \times 2$ contingency table for each substrate characterized by true-positive (tp), false-positive (fp), true-negative $(\mathrm{tn})$, and false-negative (fn) samples.

True prevalence $(\operatorname{TrP})$, apparent prevalence (ApP), sensitivity (se), specificity (sp), positive predictive value (PPV), negative predictive value (NPV), positive likelihood ratio (PLR) and negative likelihood ratio (NLR) were calculated basis on the $2 \times 2$ contingency table. The equations for calculating these parameters and their terminology are described in Thrusfield [28].

\section{Results}

The population of L. monocytogenes and E. coli O157:H7 was below enumeration limit in all samples. However, after selective enrichments and verification tests, both pathogens were detected in one strawberry sample each resulting in $3.8 \%$ prevalence for each pathogen. In Table 1 , the results obtained from each substrate utilized after the verification tests were performed, are exhibited. Regarding L monocytogenes both substrates provided with the same results, both detected the positive sample and produced no false positive or negative results. However, this was not the case as far as detection of E. coli O157:H7 was concerned. In that case, both substrates detected the true positive sample, but also detected a number of false positive ones. In Table 2 the performance indices of the substrates used are presented. In the case of ALOA and RAPID'L.mono all indices were identical since both substrates provided with the same results. In the case of E. coli $\mathrm{O} 157: \mathrm{H} 7$
Table 1. The $2 \times 2$ contingency table presenting true-positive (tp), false-positive (fp) true-negative (tn), and false-negative (fn) results for each selective medium used for the detection of L. monocytogenes or E. coli $\mathrm{O} 157: \mathrm{H} 7$ in strawberry samples after combining the results of the microbiological, biochemical, and molecular testing.

\begin{tabular}{|c|c|c|c|c|}
\hline & tp & fp & tn & fn \\
\hline \multicolumn{5}{|c|}{ L. monocytogenes } \\
\hline ALOA & 1 & 0 & 25 & 0 \\
\hline RAPID'L. mono & 1 & 0 & 25 & 0 \\
\hline \multicolumn{5}{|c|}{ E. coli O157:H7 } \\
\hline Fluorocult & 1 & 13 & 12 & 0 \\
\hline CT-SMAC & 1 & 18 & 7 & 0 \\
\hline
\end{tabular}

Table 2. Performance indices of the selective media used for the detection of $L$. monocytogenes and E. coli $\mathrm{O} 157: \mathrm{H} 7$ in strawberry samples.

\begin{tabular}{|c|c|c|c|c|}
\hline & \multicolumn{3}{|c|}{ L. monocytogenes } & \multicolumn{2}{c|}{ E. coli O157:H7 } \\
\hline & ALOA & RAPID'L. mono & Fluorocult & CT-SMAC \\
\hline TrP (\%) & \multicolumn{2}{|c|}{3.8} & \multicolumn{2}{|c|}{3.8} \\
\hline ApP (\%) & 3.8 & 3.8 & 3.8 & 3.8 \\
\hline Se (\%) & 100 & 100 & 100 & 100 \\
\hline Sp (\%) & 100 & 100 & 48 & 4 \\
\hline PPV (\%) & 100 & 100 & 7.1 & 5.3 \\
\hline NPV (\%) & 100 & 100 & 100 & 100 \\
\hline PLR & ND & ND & 1.92 & 1.04 \\
\hline NLR & 0 & 0 & 0 & 0 \\
\hline
\end{tabular}

detection, sensitivity and negative prediction value were optimal for both substrates but Fluorocult E. coli O157:H7 performed better in terms of specificity, positive prediction value and positive likelihood ratio.

Presence of L. monocytogenes in the enrichment broths was also detected by specific PCR. More accurately, in both half and full Fraser of the positive sample L. monocytogenes was detected. On the contrary, it was not detected in any of the negative samples. In the case on E. coli O157:H7, the pathogen was not detected by specific PCR in any of the enrichment broths.

\section{Discussion}

In the present study, ALOA and RAPID'L.mono exhibited identical performance. This was not the case in previous studies $[19,23,29]$ and thus it may be characterized as unusual and could probably be assigned to the rather restricted amount of samples examined in the present study. On the contrary, Fluorocult E. coli O157:H7 and CT-SMAC performance revealed their weaknesses. More accurately, the increased number of false positive results may be attributed to deviations in phenotypic traits of the isolates from what is considered as typical; at the same time the importance of the confirmatory tests is adequately highlighted. Another common characteristic of the latter two media was the absence of false negative results; thus sensitivity and negative predicting value were optimal. Sensitivity and negative predicting value are critical parameters for pathogen detection and tests exhibiting high such values are more suitable $[21,30]$.

The high number of false positive results of Fluorocult E. coli O157:H7 and CT-SMAC has also been reported by Restaino, et al. [31], Manafi and Kremsmaier [22] and Hadjilouka, et al. [19] and inevitably leads to reduced specificity, positive predicting value and positive likelihood ratio. Therefore, combination with media that provide with fewer false positive results, such as CHROMagar [32] is suggested in order to improve the accuracy of the analysis.

In the case of L. monocytogenes, the result obtained by the application of classical microbiological techniques could be predicted by specific PCR in both half and full Fraser. However, this was not 
the case for the detection of the pathogen in naturally contaminated cucumber and rocket samples, in which the inability of such an analysis to serve as an indication was highlighted [33]. Similarly, specific PCR failed to detect $E$. coli $\mathrm{O} 157: \mathrm{H} 7$ not only in the strawberry samples analyzed in the present study, but also in cucumber and rocket samples (data not shown).

\section{Conclusions}

The accuracy and reliability of L. monocytogenes and E. coli O157:H7 detection through the classical microbiological approach may be improved with parallel testing in at least two culture media. In addition, specific PCR performed after incubation of the enrichment broths is not recommended as an indicator for the presence of these pathogens.

\section{Acknowledgment}

The research leading to these results has received funding from the European Union's Seventh Framework Programme for research, technological development and demonstration under grant agreement $n^{\circ} 289719$ (Project QUAFETY: www.quafety.eu).

\section{References}

1. Bach SJ, McAllister TA, Veira DM, Gannon VPJ, Holley RA (2002) Transmission and control of Escherichia coli O157:H7-a review. Can J Anim Sci 82: 475-490.

2. Olsen SJ, Patrick M, Hunter SB, Reddy V, Kornstein L, et al. (2005) Multistate outbreak of Listeria monocytogenes infection linked to delicatessen turkey meat. Clin Infect Dis 40: 962-967.

3. Bassett J, McClure P (2008) A risk assessment approach for fresh fruits. J Appl Microbiol 104: 925-943. [Crossref]

4. WHO (2013) Enterohaemorrhagic Escherichia coli (EHEC). Media Centre. World Health Organization.

5. Galli L, Brusa V, Singh P, Cataldi AA, Manning S, et al. (2016) High prevalence of clade 8 Escherichia coli $\mathrm{O} 157: \mathrm{H} 7$ isolated from retail meat and butcher shop environment. Infect Genet Evol 45: 1-5. [Crossref]

6. Hadjilouka A, Paramithiotis S, Drosinos EH (2014) Prevalence of Listeria monocytogenes and occurrence of listeriosis from ready to eat fresh fruits and vegetables. In Hambrick EC (Ed) 'Listeria monocytogenes: Food Sources, Prevalence and Management Strategies', Nova Publishers, NY, USA, pp. 283-296.

7. Hadjilouka A, Paramithiotis S, Drosinos EH (2015) Prevalence of Escherichia coli and outbreaks from ready to eat fresh fruits and vegetables. In McCoy G (Ed) 'Coliforms: Occurrence, Detection Methods and Environmental Impact', Nova Publishers, NY, USA, pp. 71-106,

8. Beuchat LR (1998) Surface decontamination of fruits and vegetables eaten raw: a review. WHO/FSF/FOS/98.2. Food Safety Unit, World Health Organization, Geneva.

9. Little CL, Taylor FC, Sagoo SK, Gillespie IA, Grant K, et al. (2007) Prevalence and level of Listeria monocytogenes and other Listeria species in retail pre-packaged mixed vegetable salads in the UK. Food Microbiol 24: 711-717.

10. Sant'Ana AS, Igarashi MC, Landgraf M, Destro MT, Franco BDGM (2012) Prevalence, populations and pheno- and genotypic characteristics of Listeria monocytogenes isolated from ready-to-eat vegetables marketed in Sao Paulo, Brazil. Int J Food Microbiol 155: 1-9.

11. FDA (2013) Analysis and Evaluation of Preventive Control Measures for the Control and Reduction/Elimination of Microbial Hazards on Fresh and Fresh-Cut Produce. Food and Drug Administration.

12. EFSA (European Food Safety Authority), ECDC (European Centre for Disease Prevention and Control) (2010) The European Union summary report on trends and sources of zoonoses, zoonotic agents and food-borne outbreaks in 2008. EFSA Journal 8: 1496.

13. EFSA (European Food Safety Authority), ECDC (European Centre for Disease Prevention and Control) (2011) The European Union summary report on trends and sources of zoonoses, zoonotic agents and food-borne outbreaks in 2009. EFSA Journal 9: 2090.

14. EFSA (European Food Safety Authority), ECDC (European Centre for Disease Prevention and Control) (2015) The European Union Summary Report on Trends and Sources of Zoonoses, Zoonotic Agents and Food-borne Outbreaks in 2013. EFSA Journal 13: 3991.
15. EFSA (European Food Safety Authority), ECDC (European Centre for Disease Prevention and Control) (2015) The European Union Summary Report on Trends and Sources of Zoonoses, Zoonotic Agents and Food-borne Outbreaks in 2014. EFSA Journal 13: 4329.

16. HCDCP (Hellenic Center for Disease Control and Prevention) (2014) Department of Epidemiological Surveillance and Intervention. Ministry of Health. Epidemiological data for listeriosis in Greece 2004-2013.

17. EFSA (European Food Safety Authority), ECDC (European Centre for Disease Prevention and Control) (2014) The European Union summary report on trends and sources of zoonoses, zoonotic agents and food-borne outbreaks in 2012. EFSA Journal 12: 3547 .

18. HCDCP (Hellenic Center for Disease Control and Prevention) (2016) Department of Epidemiological Surveillance and Intervention. Ministry of Health. Epidemiological data of Enterohaemorrhagic E. coli infection (EHEC), Greece, 2004-2015.

19. Hadjilouka A, Mantzourani KS, Katsarou A, Cavaiuolo M, Ferrante A, et al. (2015) Estimation of Listeria monocytogenes and Escherichia coli O157:H7 prevalence and concentration in naturally contaminated rocket and cucumber samples by a deterministic and a stochastic approach. J Food Prot 78: 311-322.

20. March SB, Ratnam S (1986) Sorbitol-MacConkey medium for detection of E. coli O157:H7 associated with hemorrhagic colitis. J Clin Microbiol 23: 869-872.

21. Ottaviani F, Ottaviani M, Agosti M (1997) Differential agar medium for Listeria monocytogenes, Paper presented at the quimper froid symposium, Bretagne, France, 16-18 June.

22. Manafi M, Kremsmaier B (2001) Comparative evaluation of different chromogenic/ fluorogenic media for detecting Escherichia coli O157:H7 in food. Int J Food Microbiol 71: $257-262$.

23. Andritsos V, Mataragas M, Paramithiotis S, Drosinos EH (2013) Quantifying Listeria monocytogenes prevalence and concentration in minced pork meat and estimating performance of three culture media from presence/absence microbiological testing using a deterministic and stochastic approach. Food Microbiol 36: 395-405.

24. Jersek, T. Majstorovic, N. Klun, S.S. Mozina (2005) Impact of enrichment medium on PCR-based detection of Listeria monocytogenes in food. Acta Agric Slov 85: 15-23.

25. D'Agostino M, Wagner M, Vazquez-Boland JA, Kuchta T, Karpiskova R, et al. (2004) A validated PCR-based method to detect Listeria monocytogenes using raw milk as a food model — towards an international standard. J Food Prot 67: 1646-1655.

26. Gordillo R, Córdoba JJ, Andrade MJ, Luque MI, Rodríguez M (2011) Development of PCR assays for detection of Escherichia coli O157:H7 in meat products. Meat Sci 88 767-773. [Crossref]

27. Hadjilouka A, Andritsos ND, Paramithiotis S, Mataragas M, Drosinos EH (2014) Listeria monocytogenes serotype prevalence and biodiversity in diverse food products J Food Prot 77: 2115-2120.

28. Thrusfield M (2007) 'Veterinary epidemiology'. (3rd Edn) Blackwell Science, Oxford, UK

29. Aragon-Alegro LC, Aragon DC, Martinez EZ, Landgraf M, de Melo Franco BDG, et al. (2008) Performance of a chromogenic medium for the isolation of Listeria monocytogenes in food. Food Control 19: 483-486.

30. Stessl B, Luf N, Wagner M, Schoder D (2009) Performance testing of six chromogenic ALOA-type media for the detection of Listeria monocytogenes. J Appl Microbiol 106 651-659.

31. Restaino L, Frampton EW, Turner KM, Allison DR (1999) A chromogenic plating medium for isolating Escherichia coli O157:H7 from beef. Lett Appl Microbiol 29: 26-30. [Crossref]

32. Guali M, Ruckly C, Carle I, Lejay-Collin M, Weill FX (2013) Evaluation of CHROMagar STEC and STEC O104 chromogenic agar media for detection of Shiga toxin-producing Escherichia coli in stool specimens. J Clin Microbiol 51: 894-900.

33. Hadjilouka A, Madjourani KS, Koubou V, Paramithiotis S, Mataragas M, et al (2013) Applicability of classical and molecular techniques for the detection of L. monocytogenes in fruits and vegetables, Paper presented at the 6th European Short Course on Fresh Cut Produce Processing, Antalya, Turkey.

Copyright: (C2018 Hadjilouka A. This is an open-access article distributed under the terms of the Creative Commons Attribution License, which permits unrestricted use, distribution, and reproduction in any medium, provided the original author and source are credited. 